\title{
Contrast and spatial resolution enhancement with the transmission mode in SEM
}

Ute Golla-Schindler ${ }^{1}$, Bernd Schindler ${ }^{2}$ and Gerhard Schneider ${ }^{1}$

${ }^{1}$ 1. Materials Research Institute (IMFAA), Aalen University, Aalen, Germany, Aalen, Germany, ${ }^{2}$ Carl Zeiss Microscopy, Oberkochen, Germany, United States

The transmission mode in SEM (T-SEM) is bridging scanning electron microscopy and transmission electron microscopy. Conventionally Scanning Transmission Electron Microscopy is realized coming from the TEM side, by dedicated STEM (Scanning Transmission Electron Microscopy) or TEM/STEM, which enables the TEM and STEM mode. These instruments are operating at high accelerating voltages with lenses beyond the sample, where the aberration of the lenses beyond the sample restrict the imaging opportunities. Additionally the cross sections for elastic and inelastic scattering increase for decreasing energy of the electrons:

$\left(d \sigma_{e l} / d \Omega\right) \approx(Z / E) 2 ;\left(d \sigma_{\text {inel }} / d \Omega\right) \approx(1 / E) \quad \mathrm{E}=$ energy of the electrons $(\mathrm{keV}), \mathrm{Z}=$ atomic number

For several applications the T-SEM mode can benefit from the low electron energies and to be lens free beyond the sample. Some examples of competitive TEM and T-SEM images are shown in Figure1. First a comparison of lung cells (marked as 1) (a) $300 \mathrm{keV}$ TEM (Jeol3010), (b) $5 \mathrm{keV} \mathrm{T-SEM} \mathrm{(Zeiss} \mathrm{NVision),}$ second a comparison of a liver cell (c) $80 \mathrm{keV}$ TEM (Zeiss EM 902), (d) $8 \mathrm{keV}$ T-SEM (Hitachi S4000) and a surface layer (white arrows) on a thin carbon film (g) $80 \mathrm{keV}$ (Zeiss EM902), (i) $2 \mathrm{keV}$ T-SEM (Hitachi S4000). They confirm the significant contrast enhancement attainable in the T-SEM mode. Additionally the collection of the transmitted electrons deliver new insight in comparison to the conventional top view imaging in the SEM with backscattered (BSE) and/or secondary electrons (SE). Figs 1. (e), (f) show carbon nanotubes with Ni-particles. The contrast enhancement for the T-SEM mode enable to visualize the Ni particles and details of the carbon nanotube structure. Figs 1. (h), (i) reveal the contrast enhancement imaging a surface layer on a thin carbon layer in the SEM and T-SEM mode respectively.

Another advantage of the T-SEM mode is the decreasing interaction volume of the incoming electrons with the bulk material and, therefore, the improvement of the attainable spatial resolution. Nanoparticles (NPs) are ingredients in pharmaceutical and cosmetic products and there the precise size distribution is important for their toxicity. Figure 2. shows gold nanoparticles on a free supporting carbon film, where (a) was recorded with the Inlens SE-detector (top view) and (b) in the T-SEM mode at an accelerating voltage of $20 \mathrm{kV}$. Figs 2 (c-h) show enlarged image areas of (a) and (b), where the gold NPs and their agglomeration are detectable in both modes. The T-SEM (f, $g, h)$ mode delivers significant improvements. The NPs in area 1, 2 can be separated and the size of the NPs is not overestimated as in the top view mode (area 1, 2, 3). Region 4 shows an additionally contrast difference solely for the T-SEM image, due to the contrast enhancement in the transmission mode. All T-SEM images are recorded in the bright field mode using a combination of a conversion detector with the chamber ETD [1-3]. Benefits of this detection system is the ability to apply all available fast scan speeds especially for contaminating samples and the opportunity to work with accelerating voltages below $3 \mathrm{kV}$, where semiconductor-based systems are limited. 

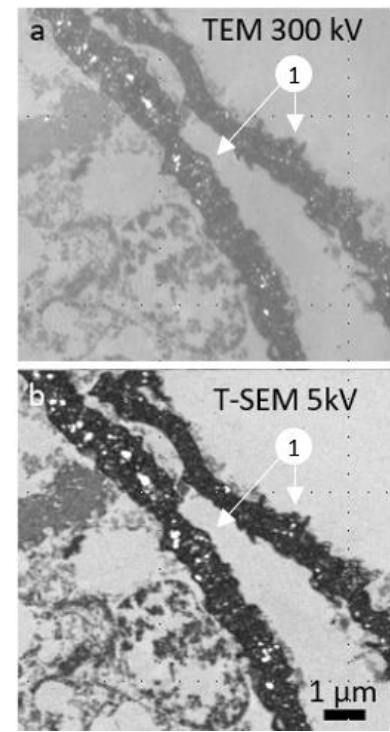
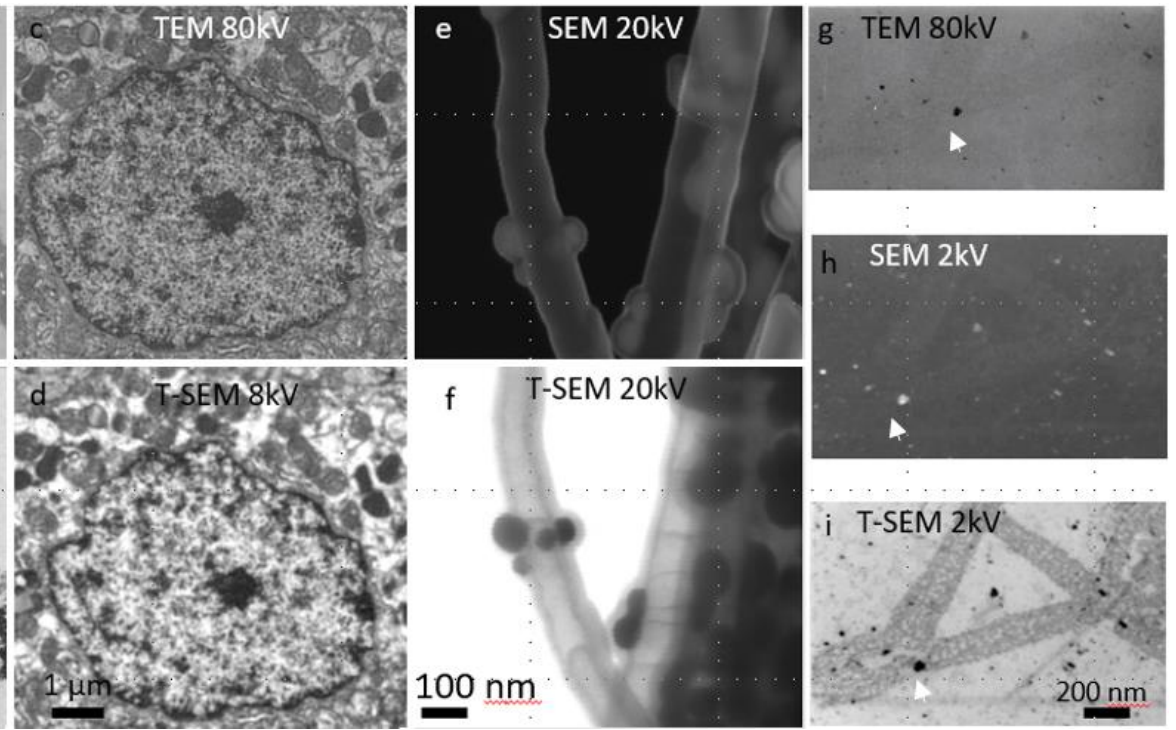

Figure 1. Contrast enhancement by the T-SEM mode. (a, b) liver cells (marked by 1) (a) TEM $300 \mathrm{kV}$, (b) T-SEM 5 kV; (c, d) liver cell (c) TEM $80 \mathrm{kV}$, d) T-SEM 8 kV; (e, f) carbon nanotubes with Ni-particles at $20 \mathrm{kV}$ (e) Inlens-SEM, (f) T-SEM; (g-i) coating on a free-supporting carbon film (g) TEM $80 \mathrm{kV}$, (h) SEM ETD $2 \mathrm{kV}$, (i) T-SEM $2 \mathrm{kV}$
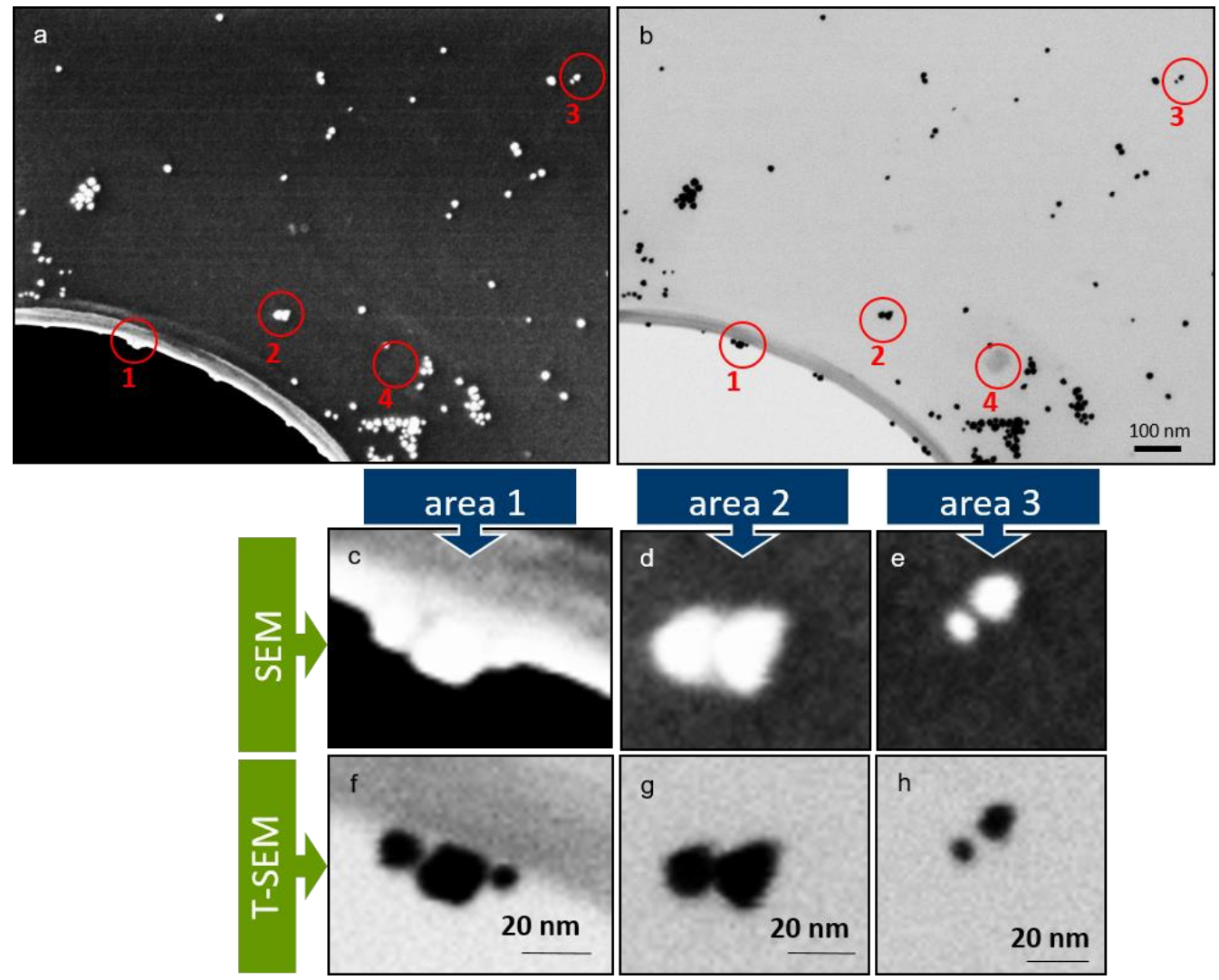

Figure 2. Gold nanoparticles on a free supporting carbon film. (a) Inlens SE image, (b) bright field image in the transmission mode collected with a conversion-detector. Enlarged image regions (areas 1, 2, 3) for (a) are shown in (c, d, e ), for (b) in (f, g, h). 


\section{References}

[1] U. Golla, B. Schindler and L. Reimer Journal of Microscopy 173 (1994)

[2] U. Golla master thesis (1993)

[3] U. Golla-Schindler (2004) STEM-Unit measurements in a scanning electron microscope. Proceedings of the 13th European Microscopy Congress. Antwerpen Vol. I 409-410 